\title{
Designing Sets of Binary Sequences for MIMO Radar Systems
}

\author{
Mohammad Alaee-Kerahroodi ${ }^{\dagger *}$ Member, IEEE, Mahmoud Modarres-Hashemi ${ }^{\ddagger}$, \\ Mohammad Mahdi Naghsh ${ }^{\ddagger}$ Member, IEEE \\ *mohammad.alaee@uni.lu
}

\begin{abstract}
In this paper, we aim at designing sets of binary sequences with good aperiodic/periodic auto- and cross-correlation functions for Multiple-Input-Multiple-Output (MIMO) radar systems. We show such a set of sequences can be obtained by minimizing a weighted sum of Peak Sidelobe Level (PSL) and Integrated Sidelobe Level (ISL) with the binary element constraint at the design stage. The sets of designed sequences are neighboring the lower bound on ISL and have a better PSL than the best known structured sets of binary sequences. To formulate the problem, we introduce a Pareto-objective of weighted auto- and cross-correlation functions by establishing a multi-objective NP-hard constrained optimization problem. Then, by using the block coordinate descent (BCD) framework, we propose an efficient monotonic algorithm based on Fast Fourier Transform (FFT), to minimize the multi-dimensional objective function. Numerical results illustrate the superior performance of the proposed algorithm in comparison with the state-of-the-art methods.
\end{abstract}

Index Terms-Aperiodic/Periodic Auto- and Cross- Correlation Functions, Binary Sets of Sequences, Block Coordinate Descent (BCD), Code-Division-Multiplexing (CDM), Integrated Sidelobe Level (ISL), Multiple-Input-Multiple-Output (MIMO), Peak Sidelobe Level (PSL), Radar, Waveform Design.

\section{INTRODUCTION}

MIMO radar systems usually radiate orthogonal (or incoherent) waveforms by their transmit antennas [1], [2] to allow matched filters separating them at receive side [3]. If the codes (waveforms) have any non-zero cross-correlation sidelobes, the energy will leak from one waveform to the other waveforms in the matched filter output [4] and this affects improperly the system performance. Hence, a successful design of orthogonal sets of sequences from a family of constant modulus discrete alphabet, with "good" auto- and cross-correlation properties is crucial for MIMO radar systems [4]-[11]. In active sensing and radar systems, due to the simplicity in waveform generation and consequently the matched filter implementation, and also in order to control the spectrum sidelobe levels of the phase changing points, sets of sequences whose entries are +1 or -1 , are typically preferred to the continuous phase sequences (which have arbitrary phases in $[0,2 \pi)$ ) [12], [13].

$\mathrm{i}^{\dagger}$ Mohammad Alaee-Kerahroodi is with the Interdisciplinary Centre for Security, Reliability and Trust (SnT), University of Luxembourg. Email: mohammad.alaee@uni.lu. The research of Mohammad Alaee-Kerahroodi leading to this paper was undertaken during his Ph.D. at the Department of Electrical and Computer Engineering, Isfahan University of Technology, Isfahan, Iran.

$\mathrm{i}^{\ddagger}$ Mahmoud Modarres-Hashemi and Mohammad Mahdi Naghsh are with the Department of Electrical and Computer Engineering, Isfahan University of Technology, Isfahan 84156-83111, Iran. Email: modarres@cc.iut.ac.ir, mm_naghsh@cc.iut.ac.ir.
Such sets of sequences, provided that have small auto-and cross-correlation sidelobes, are intrinsically suited for both separation of signals from noise and discrimination of the waveforms at output of the matched filter.

Let us consider a MIMO radar system with $N_{T}$ transmit antennas. Each antenna transmits a code vector which is composed of $N$ sub-pulses (intra-pulse coding) and can be written at the $m$-th transmit antenna as,

$$
\boldsymbol{x}_{m}=\left[x_{m}(1), x_{m}(2), \ldots, x_{m}(N)\right]^{T} \in \mathbb{C}^{N},
$$

where $x_{m}(n)$ is the $n$-th sub-pulse of the transmit code vector $\boldsymbol{x}_{m}$. Let $\left\{\boldsymbol{x}_{m}\right\}_{m=1}^{N_{T}}$ be columns of the code matrix $\boldsymbol{X}$, viz., $\boldsymbol{X}=\left[\boldsymbol{x}_{1}, \boldsymbol{x}_{2}, \ldots, \boldsymbol{x}_{N_{T}}\right] \in \mathbb{C}^{N \times N_{T}}$. The aperiodic crosscorrelation [7] of $\left\{x_{m}(n)\right\}_{n=1}^{N}$ and $\left\{x_{l}(n)\right\}_{n=1}^{N}$ at lag $k$ is defined as,

$$
r_{m l}^{\mathcal{A P}}(k)=\sum_{n=1}^{N-k} x_{m}(n) x_{l}^{*}(n+k)=r_{l m}^{\mathcal{A P} *}(-k),
$$

where $m, l=1, \ldots, N_{T},-N+1 \leq k \leq N-1$. Assuming $m=l$, equation (2) becomes the aperiodic auto-correlation of $\left\{x_{m}(n)\right\}_{n=1}^{N}$. Similarly, we define periodic cross-correlation of $\left\{x_{m}(n)\right\}_{n=1}^{N}$ and $\left\{x_{l}(n)\right\}_{n=1}^{N}$ at lag $k$ as,

$$
r_{m l}^{\mathcal{P}}(k)=\sum_{n=1}^{N} x_{m}(n) x_{l}^{*}(n+k)_{\bmod (N)}=r_{l m}^{\mathcal{P} *}(-k),
$$

where $m, l=1, \ldots, N_{T},-N+1 \leq k \leq N-1$ and (.)* denotes complex conjugate. Again, when $m=l, r_{m l}^{\mathcal{P}}(k)$ becomes the periodic auto-correlation function of $\left\{x_{m}(n)\right\}_{n=1}^{N}$. Notice that, the in-phase lag (i.e., $k=0$ ) of periodic/aperiodic auto-correlation functions represents the energy component of the sequence whereas the out-of-phase lags (i.e., $k \neq 0$ ) represent the sidelobes.

Two commonly metrics used to evaluate the goodness of the auto- and cross-correlation properties of the code vectors $\left\{\boldsymbol{x}_{m}\right\}_{m=1}^{N_{T}}$ are the PSL and the ISL which are defined as [5], [7], [9],

$$
\begin{gathered}
\mathrm{PSL}=\max \left\{\max _{m} \max _{k \neq 0}\left|r_{m m}(k)\right|, \max _{\substack{m, l \\
m \neq l}} \max _{k}\left|r_{m l}(k)\right|\right\}, \quad(4) \\
\mathrm{ISL}=\sum_{m=1}^{N_{T}} \sum_{\substack{k=-N+1 \\
k \neq 0}}^{N-1}\left|r_{m m}(k)\right|^{2}+\sum_{\substack{m, l=1 \\
m \neq l}}^{N_{T}} \sum_{k=-N+1}^{N-1}\left|r_{m l}(k)\right|^{2},
\end{gathered}
$$

where $r_{m m}(k)=r_{m m}^{\mathcal{A P}}(k)$ and $r_{m l}(k)=r_{m l}^{\mathcal{A P}}(k)$ address the aperiodic auto- and cross- correlation functions, respectively, 
whereas $r_{m m}(k)=r_{m m}^{\mathcal{P}}(k)$ and $r_{m l}(k)=r_{m l}^{\mathcal{P}}(k)$ address the periodic case ${ }^{1}$.

Note also that the correlation properties of sequences in a set play a major part in the waveform design for code division multiple access (CDMA) and wireless communication systems. In these systems, both the level of multiple access interference and code acquisition properties can be affected by the properties. In fact, the former is affected by the cross-correlation properties between different codes of the sets whereas the latter is influenced by the autocorrelation properties, that is the correlation between timeshifted versions of the same code [16]-[20]. In wireless communication systems, orthogonal sequences have been used to achieve communication among users and simultaneously manage interference as well as multiple access [21]-[23]. In this respect, structured binary sequences, like Legendre, Gold, Kasami, $m$-sequences, Gong, Paterson and Weil have been introduced and used in many CDMA/wireless communication systems [24]-[32]. However, these families are not perfectly matched with the MIMO radar requirements.

\section{A. Background and Related Works}

In order to achieve waveform orthogonality in MIMO radar systems, several approaches, including frequency-divisionmultiplexing (FDM) [33]-[35], Doppler-division multiplexing (DDM) [34], [36], [37], time-division-multiplexing (TDM) [38]-[41] and code-division-multiplexing (CDM) [4], [15] have been developed in the literature. Among them, FDM, DDM, and TDM can provide almost perfect orthogonality. However, comparing with CDM, they suffer from strong azimuth-Doppler coupling, lower amount of maximum Doppler frequency and shorter target detection range, respectively [42].

In CDM-MIMO radar systems, the requirement is to design sets of sequences with small PSL and ISL to be able to separate the transmitted waveforms from any other members of the sets at any time shift; and also to avoid masking weak targets within the range sidelobes of a strong target (see [4], [6], [9], [11], [30], [31], [43]-[47], and references therein). Unfortunately, neither the well-known Barker codes (known up to length 13) [48], nor minimum peak sidelobe (MPS) codes [49] (known up to length 105), $m$-sequences, Gold or Kasami sequences (defined for the specific lengths of $2^{n}-1$ when $n$ is a natural number) [50], which are prevalent in single-input-single-output (SISO) radar systems [51], have good properties in terms of auto/cross-correlation functions. Consequently, in recent years, a large number of researches has been devoted to design sets of sequences for MIMO radar systems, to enhance transmit beamforming [52][56], improve target detection performance [57]-[60], promote radar spatial resolution [6], [7], [24], [30], [61]-[66] and obtain better target classification/recognition performance [67]-[69]. The constraint sets considered in the design stage typically

\footnotetext{
${ }^{1}$ Notice that the Periodic Total Squared Correlation (PTSC) [14] and the Aperiodic Total Squared Correlation (ATSC) [15] are equivalent with the periodic and aperiodic ISL above, respectively.
}

are the energy, Peak-to-Average-power ratio (PAR), constant modulus and discrete/binary phase.

In this respect, Multi-CAN/Multi-PeCAN [4], [5], MM-Corr [7], ISLNew [8], [9] and Iterative Direct Search [10] algorithms are proposed to design orthogonal sets of sequences, minimizing the ISL metric (see (5)). Interestingly, all the above mentioned algorithms almost meet the lower bound on ISL [70], [71], but when designing sets of sequences with arbitrary(continuous) phases.

\section{B. Contributions}

In this paper, we consider designing sets of sequences with good aperiodic/periodic correlation properties. We consider a weighted sum of PSL and ISL as the design metric. The resulting design problem is non-convex and we devise an effective method based on BCD to tackle it. We numerically show that the sets of binary sequences that are designed with the algorithm proposed in this paper are neighborhood to the available lower bounds [70], [71], indicating its superior performance. Precisely, the major contributions of this paper can be summarized as:

- To the best of our knowledge, no mathematical algorithm with ensured convergence properties has been suggested in the literature for the exact PSL minimization over sets of sequences and so this paper is the pioneer in this aspect.

- This paper fills the relevant gap of the design problem for the set of sequences with good PSL/ISL with discrete phase property, specifically for the binary and QPSK cases. In fact, the current paper lays the ground to employ the designed binary sets in MIMO radars and communication systems with an acceptable level of implementation complexity.

Notice that, the problem of designing just one sequence with good aperiodic auto-correlation function is addressed in [12] for the case of SISO radar systems. However, the current paper deals with sets of sequences for periodic as well as aperiodic cases. Also, this paper takes into account cross-correlation functions of the sequences for the various lags to be applicable in MIMO radar systems.

\section{Organization and Notation}

The rest of this work is organized as follows. In Section II, the design problem is formulated. In Section III, we develop the BCD framework to deal with the problem. In Section IV, the solution to the scalar sub-problem for each iteration of $\mathrm{BCD}$ is derived. Section V provides several numerical experiments to verify the effectiveness of the proposed algorithm. Finally, Section VI concludes the paper.

The following notation is adopted in the paper. Bold lowercase letters for vectors and bold uppercase letters for matrices. The transpose, the conjugate, and the conjugate transpose operators are denoted by the symbols $(\cdot)^{T},(\cdot)^{*}$ and $(\cdot)^{H}$ respectively. The letter $\mathrm{J}$ represents the imaginary unit (i.e., $\jmath=\sqrt{-1}$ ). For any $x \in \mathbb{C},|x|$ and $\arg (x)$ represent the modulus and the argument of $x$, respectively. The $n$-th element of the vector $\boldsymbol{x}$ 
is denoted by $x(n)$. The abbreviation "s.t." stands for "subject to" and $\mathcal{F}_{L}($.$) illustrates the L$-points discrete-time Fourier transform.

\section{PRoblem Formulation}

In this section, we cast the code design problem to obtain sets of sequences with small out-ofphase auto-correlation and also cross-correlation values. Let $\widetilde{f}_{1}(\boldsymbol{X})=\max _{1 \leq m \leq N_{T}} \max _{k \neq 0}\left|r_{m m}(k)\right|$, $\widetilde{f}_{2}(\boldsymbol{X})=\max _{\substack{m, l \\ m \neq l}} \max _{k}\left|r_{m l}(k)\right|$ be the maximum auto- and cross-correlation sidelobes/values of all $N_{T}$ sequences, respectively. Also $\widetilde{f}_{3}(\boldsymbol{X})=\sum_{m=1}^{N_{T}} \sum_{\substack{k=-N+1 \\ k \neq 0}}^{N-1}\left|r_{m m}(k)\right|^{2}$, and $\widetilde{f}_{4}(\boldsymbol{X})=\sum_{\substack{m, l=1 \\ m \neq l}}^{N_{T}} \sum_{k=-N+1}^{N-1}\left|r_{m l}(k)\right|^{2}$ be the summation of the auto and cross-correlation sidelobes/values for all $N_{T}$ sequences, respectively. We aim at designing good sets of sequences $\boldsymbol{X}^{\star}$, minimizing $\left\{\widetilde{f}_{i}(\boldsymbol{X})\right\}_{i=1}^{4}$. The design problem is constrained to the families of constant modulus discrete phase alphabet, i. e., the $n$-th subpulse at the $m$-th transmit antenna is,

$$
x_{m}(n)=e^{\jmath \phi_{m}(n)}, \quad m=1, \ldots, N_{T} \text { and } n=1, \ldots, N
$$

with $\phi_{m}(n)$ being the phase of the $n$-th subpulse of the transmit code vector $\boldsymbol{x}_{m}$. The phase $\phi_{m}(n)$ can only be selected from the following set:

$$
\phi_{m}(n) \in\left\{0, \frac{2 \pi}{L}, \ldots, \frac{(L-1) 2 \pi}{L}\right\} \triangleq \phi_{L}
$$

where $L$ is the number of distinct phase values (e. g., $L=2$ shows the binary case and $L=4$ is the QPSK). Let $\bar{\omega}=e^{\jmath \frac{2 \pi}{L}}$ and $\Psi_{L}=\left\{1, \bar{\omega}, \ldots, \bar{\omega}^{L-1}\right\}$, then, the feasibility region for the constrained code design problem is given by,

$$
\Omega_{L}=\left\{\boldsymbol{x}_{m} \mid x_{m}(n) \in \Psi_{L}, n=1, \ldots, N\right\} .
$$

Therefore, the design problem can be cast as,

$$
P_{\boldsymbol{X}}=\left\{\begin{array}{cl}
\min _{\boldsymbol{X}} & \tilde{f}_{1}(\boldsymbol{X}), \tilde{f}_{2}(\boldsymbol{X}), \tilde{f}_{3}(\boldsymbol{X}), \tilde{f}_{4}(\boldsymbol{X}) \\
\text { s.t. } & \boldsymbol{x}_{m, l} \in \Omega_{L}, m, l=1, \ldots, N_{T}
\end{array}\right.
$$

which is a multi-objective non-convex constrained optimization problem.

In a multi-objective optimization problem, usually a feasible solution that minimizes all the objective functions simultaneously does not exist [72]. A viable means to handle these type of problems, is to use the scalarization technique ${ }^{2}$ which exploits as objective a specific weighted sum between the objective functions (Pareto-optimization) ${ }^{3}$. Particularly, a scalarization of (9) is,

$\tilde{f}_{w}(\boldsymbol{X})=w_{1} \max \left\{\tilde{f}_{1}(\boldsymbol{X}), \tilde{f}_{2}(\boldsymbol{X})\right\}+w_{2} \tilde{f}_{3}(\boldsymbol{X})+w_{3} \tilde{f}_{4}(\boldsymbol{X})$,

\footnotetext{
${ }^{2}$ Scalarizing a multi-objective problem involves the solution of conventional optimization problems whose objective function is a specific convex combination of the original figures of merits [73].

${ }^{3}$ Notice that, for the Pareto-optimization, we can consider any strictly increasing function of the original objectives.
}

where the weighting coefficients $w_{1}, w_{2}, w_{3} \in[0,1]$ and $\sum_{i=1}^{3} w_{i}=1$. The scalarization leads to the following design problem,

$$
P_{\boldsymbol{X}}^{w}= \begin{cases}\min _{\boldsymbol{X}} & \tilde{f}_{w}(\boldsymbol{X}) \\ \text { s.t. } & \boldsymbol{x}_{m, l} \in \Omega_{L}, m, l=1, \ldots, N_{T}\end{cases}
$$

where the parameters $w_{i}, i=1,2,3$ trades-off ISL and PSL values of the designed set of sequences. Precisely, $w_{1}=1$, $w_{2}=w_{3}=0$ lead to the problem of PSL minimization, whereas $w_{1}=0, w_{2}=w_{3}=0.5$ correspond to the ISL minimization problem. In fact, the different values of $w_{i}$ can lead to various sets of sequences with similar goodness, which is an important property for the next-generation MIMO radar systems. An example is automotive MIMO radar systems, where the performance can be enhanced through a continuous and coordinated feedback between the transmitter and receiver which implies a dynamic adaptation of the sensor's algorithms to the operational context and environmental replies [74], [75]. In this case, this is possible to choose $w_{i}$ according to the environment, whether we face with an extended target (low ISL waveforms are desired) or we are in dense target scene (low PSL waveforms are desired).

\section{The Proposed Method}

This section introduces an iterative derivative-free optimization algorithm, based on the BCD minimization procedure, by updating just one or a few blocks of variables at a time, rather than updating all the blocks together (the batch update) [76]. Indeed, to handle the minimization problem in (11), we need to loop over all the coordinates and resort to the following subproblems:

- Outer loop; Pick a coordinate $t$ from $1, \ldots, N_{T}$ and design a code vector $\boldsymbol{x}_{t}$ keeping the other code vectors fixed.

- Inner loop; Pick a coordinate $d=1, \ldots, N$ in the selected coordinate $t$ to optimize each scalar variable $x_{t}(d)$ of $\boldsymbol{x}_{t}$, keeping fixed the other entries of the code vector $\boldsymbol{x}_{t}$.

Therefore, by solving a sequence of simpler optimization problems, each subproblem will have a lower dimension in the minimization procedure, and thus can typically be solved easier than the original problem. The general idea to tackle $P_{\boldsymbol{X}}^{w}$ is summarized below:

1) Pick coordinate $t$ from $1,2, \ldots, N_{T}$.

2) Set $\boldsymbol{x}_{t}^{(i+1)}=\arg \min _{\boldsymbol{x}_{t}} \widetilde{f}_{w}\left(\boldsymbol{x}_{t}, \boldsymbol{X}_{-t}^{(i)}\right)$.

where $\boldsymbol{X}_{-t}^{(i)}$ represents all other coordinates which are kept fixed during the iteration $(i+1)$ of the outer loop, i.e.,

$\boldsymbol{X}_{-t}^{(i)}=\left[\boldsymbol{x}_{1}^{(i)}, \boldsymbol{x}_{2}^{(i)}, \ldots, \boldsymbol{x}_{t-1}^{(i)}, \boldsymbol{x}_{t+1}^{(i)}, \ldots, \boldsymbol{x}_{N_{T}}^{(i)}\right] \in \mathbb{C}^{N \times N_{T}-1}$.

Accordingly, in the outer loop, the optimization Problem $P_{\boldsymbol{X}}^{w}$ at iteration $(i+1)$ boils down to,

$$
P_{t, \boldsymbol{X}^{(i)}}^{w}= \begin{cases}\min _{\boldsymbol{x}_{t}} & \widetilde{f}_{w}\left(\boldsymbol{x}_{t}, \boldsymbol{X}_{-t}^{(i)}\right) \\ \text { s.t. } & \boldsymbol{x}_{t} \in \Omega_{L}, n=1, \ldots, N\end{cases}
$$


where

$\widetilde{f}_{w}\left(\boldsymbol{x}_{t}, \boldsymbol{X}_{-t}^{(i)}\right)=\widetilde{f}_{w}\left(\boldsymbol{x}_{1}^{(i)}, \boldsymbol{x}_{2}^{(i)}, \ldots, \boldsymbol{x}_{t-1}^{(i)}, \boldsymbol{x}_{t}, \boldsymbol{x}_{t+1}^{(i)}, \ldots, \boldsymbol{x}_{N_{T}}^{(i)}\right)$.

Thus, denoting by $\boldsymbol{X}_{t}^{\star(i+1)}$ the optimal solution to $P_{t, \boldsymbol{X}^{(i)}}^{w}$, the optimized code matrix at iteration $(i+1)$ becomes,

$$
\boldsymbol{X}_{t}^{\star(i+1)}=\left[\boldsymbol{x}_{1}^{(i)}, \boldsymbol{x}_{2}^{(i)}, \ldots, \boldsymbol{x}_{t-1}^{(i)}, \boldsymbol{x}_{t}^{\star}, \boldsymbol{x}_{t+1}^{(i)}, \ldots, \boldsymbol{x}_{N_{T}}^{(i)}\right] .
$$

Further, to obtain the optimal code entry $x_{t}(d)$, we undertake the following steps:

- Pick coordinate $d$ from $1,2, \ldots, N$.

- Set $x_{t}^{(h+1)}(d)=\arg \min _{x_{t}(d)} g_{w, \boldsymbol{X}_{-t}^{(i)}}\left(x_{t}(d), \boldsymbol{x}_{t,-d}^{(h)}\right)$.

where $\boldsymbol{x}_{t,-d}^{(h)}$ represents all other coordinates of the code vector $\boldsymbol{x}_{t}$ which are keeping fixed at iteration $(h+1)$ of the inner loop ${ }^{4}$ and $g_{w, \boldsymbol{X}_{-t}^{(i)}}\left(x_{t}(d), \boldsymbol{x}_{t,-d}^{(h)}\right)$ is introduced shortly. Indeed, in the inner loop, we go through the $t$-th selected block and choose the scalar $x_{t}(d)$ as the variable to be optimized, put the remaining code entries at iteration $(h+1)$ in the vector $\boldsymbol{x}_{t,-d}^{(h)} \in \mathbb{C}^{N-1}$ defined as,

$\boldsymbol{x}_{t,-d}^{(h)}=\left[x_{t}^{(h)}(1), \ldots, x_{t}^{(h)}(d-1), x_{t}^{(h)}(d+1), \ldots, x_{t}^{(h)}(N)\right]^{T}$.

Now, the resulting optimization problem is given by,

$$
P_{d, \boldsymbol{x}_{t}^{(h)}}^{w} \begin{cases}\min _{x_{t}(d)} & g_{w, \boldsymbol{X}_{-t}^{(i)}}\left(x_{t}(d) ; \boldsymbol{x}_{t,-d}^{(h)}\right) \\ \text { s.t. } & x_{t}(d) \in \Omega_{L}\end{cases}
$$

where $^{5}$

$$
g_{w, \boldsymbol{X}_{-t}^{(i)}}\left(x_{t}(d) ; \boldsymbol{x}_{t,-d}^{(h)}\right)=w_{1} g_{1}+w_{2} g_{2}+w_{3} g_{3}
$$

with $^{6}$

$$
g_{1}=\max \left\{\max _{k \neq 0}\left|r_{t t}(k)\right|, \max _{\substack{l \\ l \neq t}} \max _{k}\left|r_{t l}(k)\right|\right\},
$$

and

$$
g_{2}=\sum_{\substack{k=-N+1 \\ k \neq 0}}^{N-1}\left|r_{t t}(k)\right|^{2}, \quad g_{3}=\sum_{\substack{l=1 \\ l \neq t}}^{N_{T}} \sum_{k=-N+1}^{N-1}\left|r_{t l}(k)\right|^{2} .
$$

Therefore, the optimized code vector at the $t$-th transmit antenna is,

$$
\boldsymbol{x}_{t}^{(h+1)}=\left[x_{t}^{(h)}(1), x_{t}^{(h)}(2), \ldots, x_{t}^{\star}(d), \ldots, x_{t}^{(h)}(N)\right]^{T},
$$

where $x_{t}^{\star}(d)$ is the solution to (16). As a result, starting from an initial code matrix $\boldsymbol{X}^{(0)}$, the code matrices $\boldsymbol{X}^{(1)}$, $\boldsymbol{X}^{(2)}, \boldsymbol{X}^{(3)}, \ldots$ are obtained iteratively. A summary of the proposed approach to obtain $\boldsymbol{X}^{\star}$ can be found in Algorithm 1. Since the proposed approach can effectively design binary

\footnotetext{
${ }^{4}$ The super scripts $(i)$ and $(i+1)$ for $\boldsymbol{x}_{t}$ and $\boldsymbol{x}_{t}^{\star}$ is implicit and omitted for simplicity.

${ }^{5}$ In Appendix A, we provide an explicit dependency of $g_{w, \boldsymbol{X}_{-t}^{(i)}}\left(x_{t}(d) ; \boldsymbol{x}_{t,-d}^{(h)}\right)$ on the optimization variable $x_{t}(d)$.

${ }^{6}$ Note that the dependency of $g_{i}, i=1,2,3$ on $w$, and $\boldsymbol{X}_{-t}^{(i)}$ is implicit and omitted due to the simplicity. Also, the dependency of $r_{t t}(k)$ and $r_{t l}(k)$ on $x_{t}(d)$ is implicit.
}

set of sequences, we name it Binary Sequences seTs $(\text { BiST) })^{7}$ method. After initialization by $\boldsymbol{X}^{(0)}$, various code vectors $\left\{\boldsymbol{x}_{t}\right\}_{t=1}^{N_{T}}$ are optimized sequentially in step (2) of Algorithm 1 till convergence. The superscript $(i)$ shows iterations on the mentioned code vector (outer loop). When the code vector $\boldsymbol{x}_{t_{0}}$ is selected, we resort to Algorithm 2, to obtain the optimized vector $\boldsymbol{x}_{t_{0}}^{\star}$. In Algorithm 2, each entry of $\boldsymbol{x}_{t_{0}}$ is optimized via CD technique. Herein, the superscript $(h)$ denotes iterations for optimizing various entries in $\boldsymbol{x}_{t_{0}}$; i. e., $h$ varies from 0 to $N-1$ (number of correlation lags) at every call to Algorithm 2 (inner loop). Finally, when an entry $\boldsymbol{x}_{t_{0}}\left(d_{0}\right)$ of the code $\boldsymbol{x}_{t_{0}}$ is selected, the associated scalar optimization Problem $P_{d, \boldsymbol{x}_{t}^{(h)}}^{w}$ is solved via Algorithm 3 to be discussed in the next section. Note that Algorithm 2 terminates when all entries of $\boldsymbol{x}_{t_{0}}$ are optimized ( $h=N-1)$, whereas Algorithm 1 is considered to be converged when the iterative change in the objective of Problem $P_{\boldsymbol{X}}^{w}$ is practically small.

Remark 1. The proposed method decreases the value of the objective function in (11), i. e., $\widetilde{f}_{w}(\boldsymbol{X})$, at each iteration, and can ensure convergence to a stationary point provided that the Maximum Block Improvement (MBI) [77], [78] rule is adopted. However, we numerically observed similar performances for Algorithm 1 and the case of applying a full CD, using the MBI rule (ensuring the convergence to a stationary point). Notice that, the convergence speed/run-time is more when applying a full $C D$ using the MBI rule.

\begin{tabular}{l}
\hline Algorithm 1 BiST algorithm for MIMO radar systems \\
\hline Input: Initial code matrix $\boldsymbol{X}^{(0)} \in \mathbb{C}^{N \times N_{T}}$, threshold $\epsilon>0$,
\end{tabular} $w_{i} \in[0,1]$, provided that $\sum_{i=1}^{3} w_{i}=1$;

Output: Optimized sequence set $\boldsymbol{X}^{\star}$;

\section{1) Initialization.}

- Compute the initial objective value $\widetilde{f}_{w}\left(\boldsymbol{x}_{1}^{(0)}, \boldsymbol{x}_{2}^{(0)}, \ldots, \boldsymbol{x}_{N_{T}}^{(0)}\right)$;

- Set $t:=1$ and $i:=0$;

2) Improvement.

- Use Algorithm 2 to solve $P_{t, \boldsymbol{X}^{(i)}}^{w}$, and obtain $\boldsymbol{x}_{t}^{\star}$;

- Set $i:=i+1$ and $\boldsymbol{X}_{t}^{(i)}=\left[\boldsymbol{x}_{1}^{(i-1)}, \boldsymbol{x}_{2}^{(i-1)}, \ldots, \boldsymbol{x}_{t}^{\star}, \ldots, \boldsymbol{x}_{N_{T}}^{(i-1)}\right] ;$

3) Stopping Criterion.

- If $\left|\widetilde{f}_{w}\left(\boldsymbol{X}_{t}^{(i)}\right)-\widetilde{f}_{w}\left(\boldsymbol{X}_{t}^{(i-1)}\right)\right|<\epsilon$, stop. Otherwise, update $t$, i.e., if $t<N_{T}, t=t+1$, otherwise $t=1$, and go to the step (2);

4) Output.

- Set $\boldsymbol{X}_{t}^{\star}=\boldsymbol{X}_{t}^{(i)}$.

\section{The Code Entry Design}

The devised method for dealing with the design Problem $P_{\boldsymbol{X}}^{w}$ was presented in Section III. Now, we consider the optimization (16), i. e., the problem associated with optimizing the scalar entry $x_{t}(d)$ of the code vector $\boldsymbol{x}_{t}$.

${ }^{7}$ BiST means the number " 20 " in Persian language and also refers to something that is perfect. 


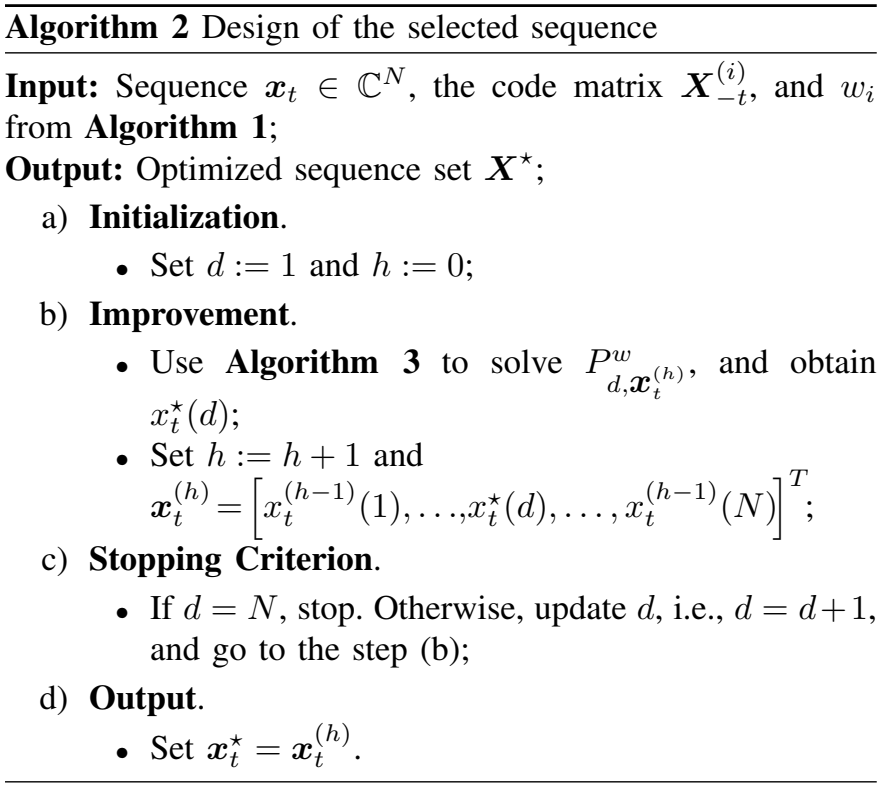

In terms of the phase variable $\phi_{t_{d}}=\arg \left(x_{t}(d)\right)$, the optimization Problem $P_{d, \boldsymbol{x}_{t}^{(h)}}^{w}$ can be expressed as Problem $P_{d, \phi_{t_{d}}^{(h)}}^{w}$ in (18) at top of the next page. In the sequel, we propose an algorithm to globally find the solution to Problem $P_{d, \boldsymbol{x}_{t}^{(h)}}^{w}$.

A) The Binary Case $(L=2)$

It can be shown that the aperiodic/periodic crosscorrelation lags in correspondence of the phase variable $\phi_{t_{d}}=\arg \left(x_{t}(d)\right)$ can be written as (see Appendix A),

$$
r_{t l, \phi_{t_{d}}}(k)=a_{d k l} e^{\jmath \phi_{t_{d}}}+c_{d k l} .
$$

Also, the aperiodic/periodic auto-correlation lags are given by (see Appendix A):

$$
r_{t t, \phi_{t_{d}}}(k)=a_{d k t} e^{\jmath \phi_{t}}+c_{d k t} .
$$

Note that $\phi_{t_{d}}=\pi(q-1), q=1,2$. Hence. in the following we use the notation $\phi_{t_{d}}\left(q_{0}\right)$ to show $\phi_{t_{d}}=\pi\left(q_{0}-1\right)$. Next, inspired by Discrete-Time Fourier Transform (DFT) definition, we devise an identity between DFT of the coefficients $a_{d k z}, c_{d k z}$ ( $z$ stands for either $t$ or $l$ ) and magnitude of correlation lags to be used for efficient computing of the solution to $P_{d, \boldsymbol{x}_{t}^{(h)}}^{w}$. Let

$$
\boldsymbol{\nu}_{d k z}=\left[\left|r_{t z, \phi_{t_{d}}(1)}(k)\right|,\left|r_{t z, \phi_{t_{d}}(2)}(k)\right|\right]^{T} \in \mathcal{R}^{2},
$$

and

$$
\boldsymbol{\zeta}_{d k z}=\left[a_{d k z}, c_{d k z}\right]^{T} \in \mathcal{R}^{2}
$$

observe that,

$$
\left|\mathcal{F}_{L}\left(\boldsymbol{\zeta}_{d k z}\right)\right|=\left[\left|r_{t z, k}\left(\phi_{t_{d}}(1)\right)\right|,\left|r_{t z, k}\left(\phi_{t_{d}}(2)\right)\right|\right]^{T} .
$$

Verifying above is straight forward; precisely by noting,

$$
\mathcal{F}_{L}\left(\boldsymbol{\zeta}_{d k z}\right)=\left[\begin{array}{c}
a_{d k z}+c_{d k z} \\
a_{d k z}-c_{d k z}
\end{array}\right]
$$

and,

$$
r_{t z, k}\left(\phi_{t_{d}}\right) e^{-\jmath \phi_{t_{d}}(q)}=a_{d k z}+c_{d k z} e^{-\jmath \phi_{t_{d}}(q)},
$$

and equivalently,

$$
\left|r_{t z, k}\left(\phi_{t_{d}}\right) e^{-\jmath \phi_{t_{d}}(q)}\right|=\left|r_{t z, k}\left(\phi_{t_{d}}\right)\right|
$$

which leads to (20). Hence,

$$
\nu_{d k z}=\left|\operatorname{DFT}\left(\boldsymbol{\zeta}_{d k z}\right)\right|
$$

where $\operatorname{DFT}\left(\boldsymbol{\zeta}_{d k z}\right)$ is the $L$-points DFT of the vector $\boldsymbol{\zeta}_{d k z}$. Next, we define the matrix $\boldsymbol{U}_{z} \in \mathcal{R}^{(2 N-1) \times 2}$ whose $k$-th row is $\boldsymbol{\nu}_{d k z}^{T}$. In fact, the matrix $\boldsymbol{U}_{t}$ contains modulus of all auto-correlation lags (i. e., $k$ ), whereas all the modulus values of cross-correlation lags are gathered in $\boldsymbol{U}_{l}$. Let $\boldsymbol{u}_{z}^{m} \in \mathcal{R}^{2}$ be a vector containing the maximum values of each columns of $\boldsymbol{U}_{z}$. On the other hand, let $\widetilde{\boldsymbol{u}}_{z}^{s} \in \mathcal{R}^{2}$ be a vector containing the summation of the squared values of each columns of the matrix $U_{z}$. Hence,

$$
\boldsymbol{\omega}_{t}(d)=w_{1} \max \left\{\boldsymbol{u}_{t}^{p}, \max _{\substack{l \\ l \neq t}} \boldsymbol{u}_{l}^{p}\right\}+w_{2} \widetilde{\boldsymbol{u}}_{t}^{s}+w_{3} \sum_{\substack{l=1 \\ l \neq t}}^{N_{T}} \widetilde{\boldsymbol{u}}_{l}^{s}
$$

where $\boldsymbol{\omega}_{t}(d) \in \mathcal{R}^{2}$ and the operation of maximum between two vectors is defined element wise. Then, the optimal solution to $P_{d, \phi_{t}^{(h)}}^{w}$ is given by

$$
\phi_{t_{d}}^{\star}=\pi\left(q^{\star}-1\right)
$$

where

$$
q^{\star}=\arg \min _{q=1,2}\left\{\boldsymbol{\omega}_{t}(d)\right\}
$$

and, the optimal phase code entry is, $x_{t}^{\star}(d)=e^{\jmath \phi_{t_{d}}^{\star}}$

B) General Discrete Phase ( $L \geq 3$ ). The proposed solution for $L \geq 3$ is similar to that of the binary case, but with a minor modification. More precisely, in this case it can be shown,

$$
r_{t l, \phi_{t_{d}}}(k)=a_{d k l} e^{\jmath \phi_{t}}+c_{d k l},
$$

and

$$
r_{t t, \phi_{t_{d}}}(k)=a_{d k t} e^{\jmath \phi_{t}}+b_{d k t} e^{-\jmath \phi_{t}}+c_{d k t} .
$$

In a similar manner, let $\phi_{t_{d}}(q)=\frac{2 \pi(q-1)}{L}, q=1, \ldots, L$, $\boldsymbol{\nu}_{d k z}=\left[\left|r_{t z, \phi_{t_{d}}(1)}(k)\right|,\left|r_{t z, \phi_{t_{d}}(2)}(k)\right|, \ldots,\left|r_{t z, \phi_{t_{d}}(L)}(k)\right|\right]^{T}$, where $z$ stands for either $t$ or $l$, then,

$$
\begin{gathered}
\boldsymbol{\zeta}_{d k t}=\left[a_{d k t}, c_{d k t}, b_{d k t}, \mathbf{0}_{1 \times(L-3)}\right]^{T} \in \mathcal{R}^{L}, \\
\boldsymbol{\zeta}_{d k l}=\left[a_{d k l}, c_{d k l}, \mathbf{0}_{1 \times(L-2)}\right]^{T} \in \mathcal{R}^{L} .
\end{gathered}
$$

The definition of the matrix $\boldsymbol{U}_{z}$ and the vectors $\boldsymbol{u}_{z}^{m}, \widetilde{\boldsymbol{u}}_{z}^{s}$ are similar but with an updated dimension; namely $\boldsymbol{U}_{z} \in$ $\mathcal{R}^{(2 N-1) \times L}$ and $\boldsymbol{u}_{z}^{m}, \widetilde{\boldsymbol{u}}_{z}^{s} \in \mathcal{R}^{L}$. Finally, $\phi_{t_{d}}^{\star}=\frac{2 \pi\left(q^{\star}-1\right)}{L}$. The method of designing the code entry is provided in Algorithm 3 .

Remark 2. In Algorithm 3, calculation of $\nu_{d k t}$ needs $(2 N-1)$ L-points FFTs, whereas $\nu_{d k l}$ needs $N_{T}(2 N-1) L$-points FFTs. Also Algorithm 2, runs Algorithm 3 for $N$ - times. Therefore, for each selected sequence $\boldsymbol{x}_{t}$ (i. e., each iteration in Algorithm 1), the computational complexity is 


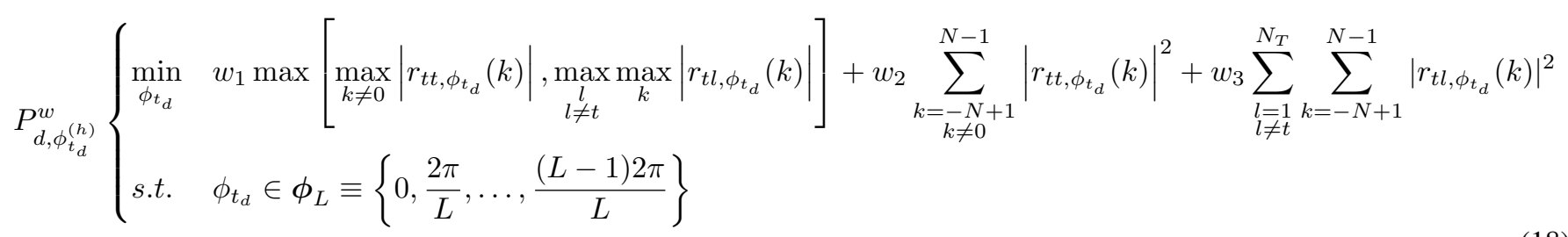

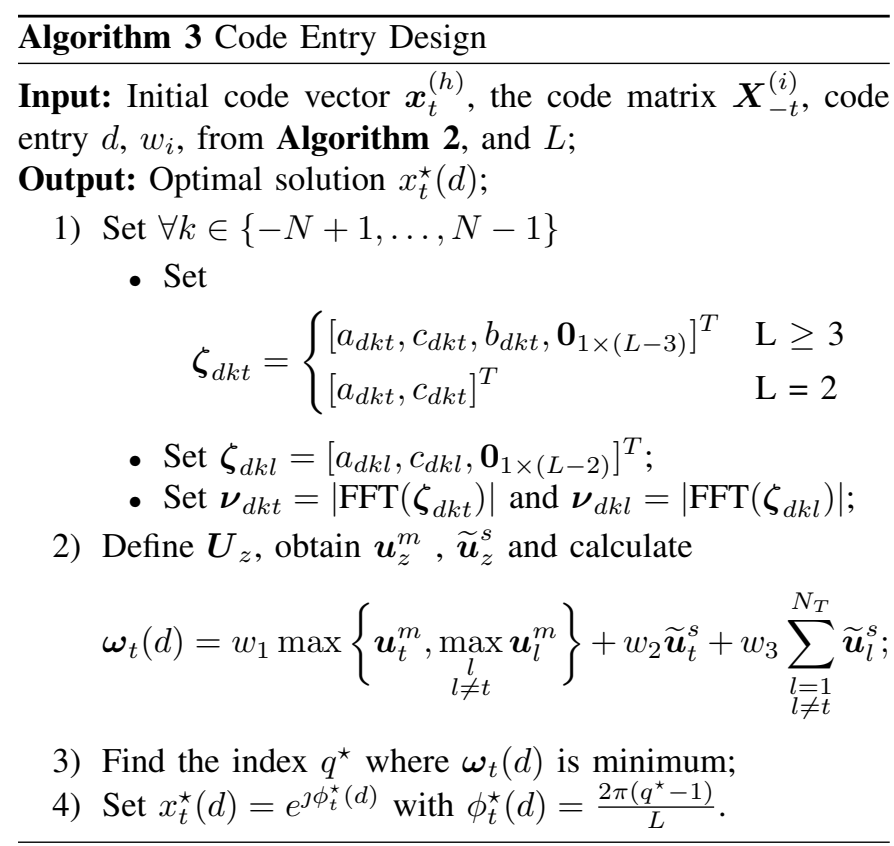

$\mathcal{O}\left(N^{2} N_{T} L \log _{2} L\right)$. Consequently, in case of designing binary sets of sequences (i.e., $L=2$ ), the overall computational complexity per iteration is $\mathcal{O}\left(N^{2} N_{T}\right)$.

\section{Performance Analysis}

In this section, we provide several numerical examples to illustrate the superior performance of the proposed method in designing binary/discrete phase sets of sequences. In order to evaluate of the performance of the proposed algorithm (BiST) via a normalized metric, we use the definitions

$$
\operatorname{ISLR}(\mathrm{dB})=10 \log _{10} \frac{\mathrm{ISL}}{N^{2}},
$$

which is the ratio of integrated energy of the sidelobes to the peak energy of the mainlobe and

$$
\operatorname{PSLR}(\mathrm{dB})=10 \log _{10} \frac{\mathrm{PSL}^{2}}{N^{2}},
$$

which is the ratio of maximum energy of the sidelobes to the peak energy of the mainlobe. Also, we set the threshold $\epsilon=10^{-5}$ and consequently the stopping criteria $\mid \widetilde{f}_{w}\left(\boldsymbol{X}^{(i)}\right)-$ $\widetilde{f}_{w}\left(\boldsymbol{X}^{(i-1)}\right) \mid \leq 10^{-5}$ is used to terminate Algorithm 1.

\section{A. ISL Minimization}

We set $w_{1}=0$, and $w_{2}=w_{3}=0.5$, number of transmit antennas $N_{T} \in\{3,4\}$ to perform ISL minimization, and adopt

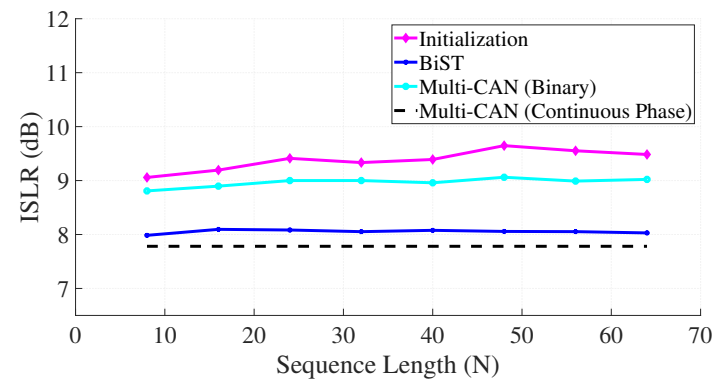

(a) $N_{T}=3$.

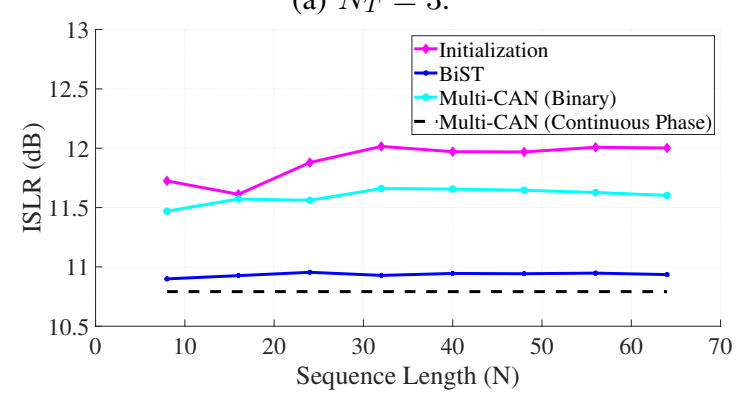

(b) $N_{T}=4$.

Fig. 1: Comparison between the averaged aperiodic ISLR values of the obtained set of sequences via BiST and MultiCAN algorithms over 10 independent trails ; a) The number of transmit antennas $N_{T}=3$, b) $N_{T}=4$.

Multi-CAN as the benchmark ${ }^{8}$. Notice that the lower bound for the ISLR is $10 \log N_{T}\left(N_{T}-1\right)$ [7]. Thus considering $N_{T}=3$, and $N_{T}=4$, the lower bounds of $7.78 \mathrm{~dB}$, and $10.79 \mathrm{~dB}$ will be obtained, respectively. In Fig. 1, the averaged aperiodic ISLR values for sets of sequences obtained via the BiST for the code lengths $N=\{8,16,24,32,40,48,56,64\}$, is depicted. The figure shows that, the obtained sets of binary sequences are neighboring to around $0.2 \mathrm{~dB}$ of the lower bound. According to this figure, BiST has provided sets of binary sequences close to the sets obtained via Multi-CAN (continuous phase-which meets the lower bound), but interestingly with $\{ \pm 1\}$ alphabets. The averaged results over 10 independent trials are reported for both Multi-CAN and BiST algorithms. Meanwhile, the averaged ISLR values (over 10 independent trials) of the initializing random sets of sequences, are also depicted in this figure. To observe the effectiveness of BiST algorithm, we also have plotted the best set of binary sequences, obtained via quantization of the Multi-CAN

\footnotetext{
${ }^{8}$ The Matlab codes for Multi-CAN algorithm are downloaded from the book website http://www.sal.ufl.edu/book/.
} 


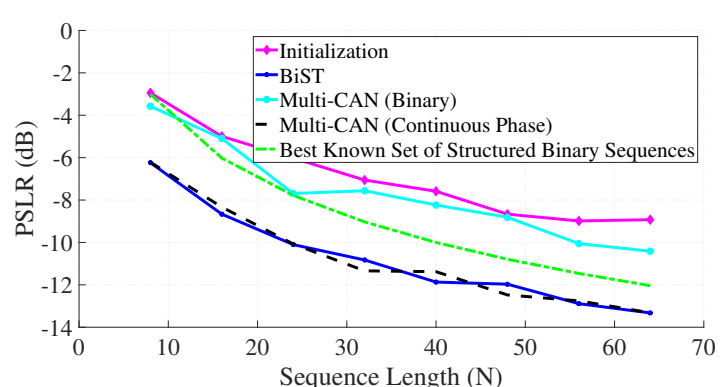

(a) $N_{T}=3$.

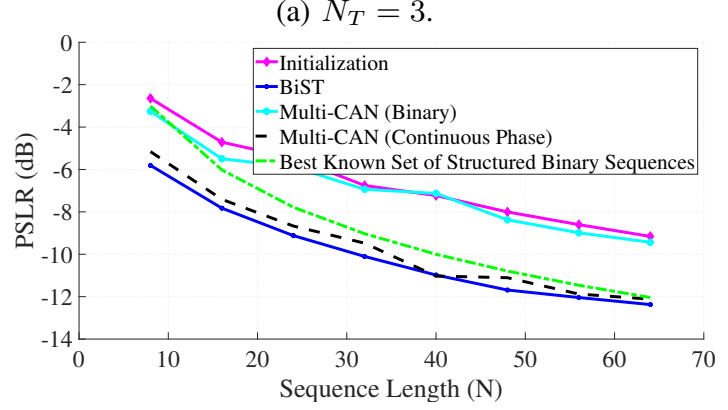

(b) $N_{T}=4$.

Fig. 2: Comparison between the averaged aperiodic PSLR values of the obtained sets of sequences via BiST and MultiCAN algorithms over 10 independent trails. The bound $2 \sqrt{N}$ provided by the best known sets of structured binary sequences is also depicted.

sequences. Of course the quantization cannot provide a good set of sequences, particularly when the alphabet size is small.

\section{B. PSL Minimization}

Herein, we consider $w_{1}=1$, and $w_{2}=w_{3}=0$, with code length $N=\{8,16,24,32,40,48,56,64\}$, and number of transmit antennas $N_{T}=3,4$ to resort to the PSL minimization. As to the best of our knowledge, there is no binary set of sequences that meets the lower bound on PSL [70]. According to the literature, best PSL values for the families of structured binary sequences ${ }^{9}$, like Legendre, Gold, Kasami, $m$-Sequences, Gong, Paterson and Weil is $2 \sqrt{N}$ (see [79] and references therein). In the simulations herein, we consider $2 \sqrt{N}$ as the PSL of the best known set of structured binary sequences as the comparison benchmark.

Fig. 2 illustrates the averaged PSLR values for both BiST and Multi-CAN, obtained over 10 independent trails. It can be seen, sets of sequences obtained via BiST have better PSLR values than the bound (i. e., $2 \sqrt{N}$ ). Also, as mentioned before, Multi-CAN designs sets of sequences with continuous phases, minimizing the ISL metric. The best sets of sequences obtained via quantization of Multi-CAN is also plotted in this figure. It can be observed from the figure that BiST has provided sets of binary sequences almost better than the set obtained via continuous phase Multi-CAN, but with $\{ \pm 1\}$ alphabet. This can be explained using the fact that

\footnotetext{
${ }^{9}$ Notice that, the structured sets of sequences (e. g. Kasami, Gold, etc.,), are constructed of specific lengths to obtain good periodic correlation functions and there still is a gap between their aperiodic PSL/ISL, and the lower bound.
}

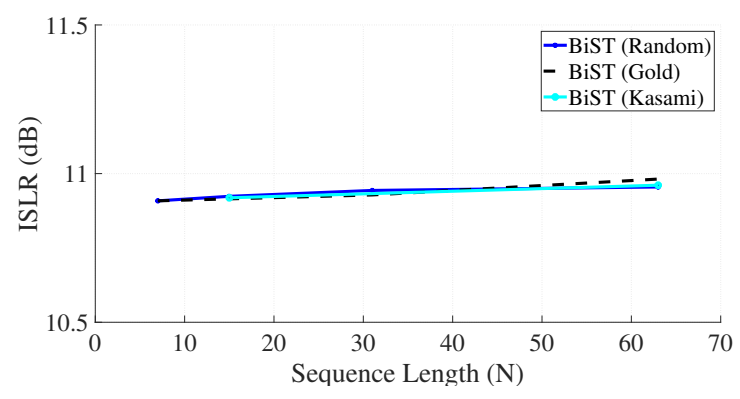

Fig. 3: Comparison between the obtained aperiodic ISLR values when starting from different sets of sequences $\left(N_{T}=4\right)$.

BiST directly minimizes the PSL. Also, the averaged PSLR values (over 10 independent trials in each sequence length) of the initializing random set of sequences and the best set of binary sequences obtained via quantization of the Multi-CAN sequences, are depicted in this figure.

\section{The Effect of the Initialization}

In order to asses the initialization effects on the performance of the proposed algorithm, we provide the numerical examples in the case of PSL minimization. Notice that, we numerically observed that for the case of ISL minimization, the proposed algorithm almost meets the lower bound and the initialization has no significant effect (see Fig. 3 below as an example). In Fig. 4, a comparison between the obtained periodic PSLR values when initialized with random, Gold and Kasami sequences is depicted. The bound $2 \sqrt{N}$ provided by the best known sets of structured binary sequences (i. e., Gold, Kasami, $m$-sequences, etc.) is also illustrated in this figure. According to Fig. 4, starting from Kasami/Gold, the obtained sets of sequences have lower (better) PSLR values. It can be due to the goodness of these sets when considering periodic auto-correlation function.

Fig. 5 depicts a similar comparison but for the aperiodic case. As this figure shows, the proposed algorithm has provided better PSLR values when it is initialized from a set of random sequences. In essence, we numerically observed no significant improvement in performance for various initialization in aperiodic PSL/ISL or periodic ISL. However, for the periodic PSL minimization, a reduction usually occurs when initializing by known sequences like Kasami.

\section{Set Size and Alphabet Size}

Next we consider the effect of the set and alphabet size. In Fig. 6, we plot aperiodic PSLR and ISLR values of obtained sets of sequences when code length $N=64$, and number of transmit antennas $N_{T}=[2,3,4,5,6,7]$. Notice that the obtained PSLR/ISLR values are averaged over 10 independent trials. The results indicate that in terms of aperiodic PSLR (Fig. 6a), the designed sets of sequences are always better than Multi-CAN (Binary) and they are better than Multi-CAN (Continuous Phase) when $N_{T} \geq 4$. Also, in terms of aperiodic ISL minimization, the obtained ISLR values in Fig. $6 \mathrm{~b}$ are close to those of Multi-CAN (Continuous Phase) which the latter meets the ISL lower bound [7], [70], [71]. 


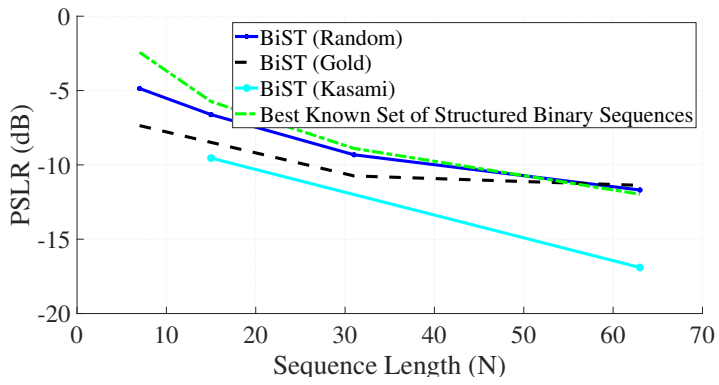

(a) $N_{T}=3$.

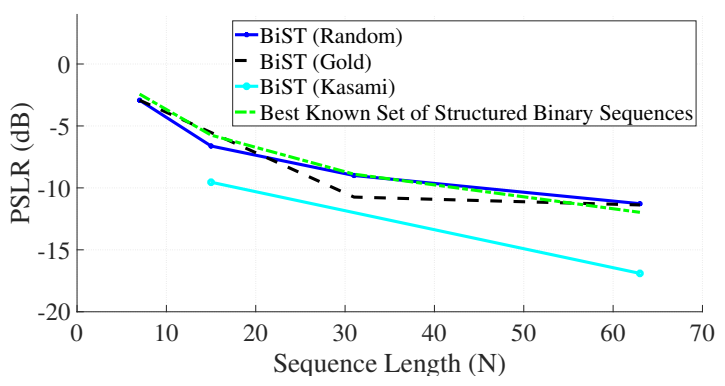

(b) $N_{T}=4$.

Fig. 4: Comparison between the obtained periodic PSLR values starting from different sets of sequences. The bound $2 \sqrt{N}$ provided by the best known sets of structured binary sequences is also included.

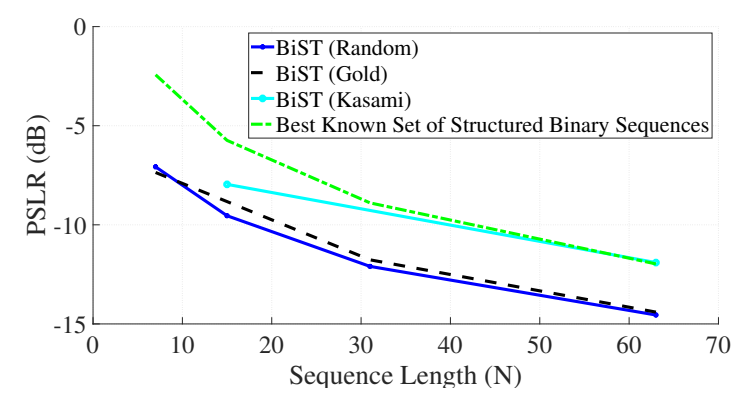

(a) $N_{T}=3$.

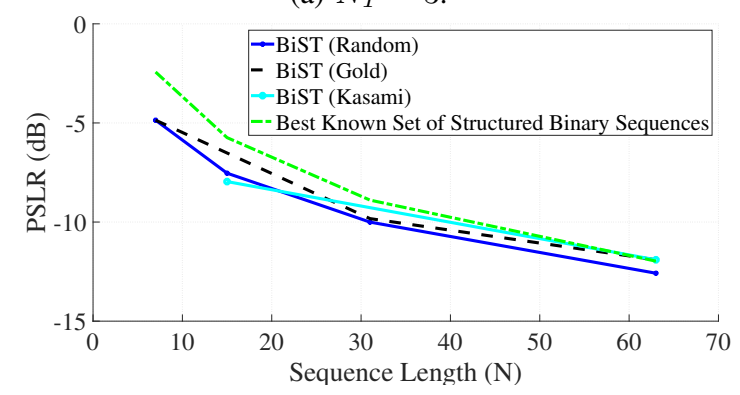

(b) $N_{T}=4$.

Fig. 5: Comparison between the obtained aperiodic PSLR values starting from different sets of sequences. The bound $2 \sqrt{N}$ provided by the best known sets of structured binary sequences is also included.

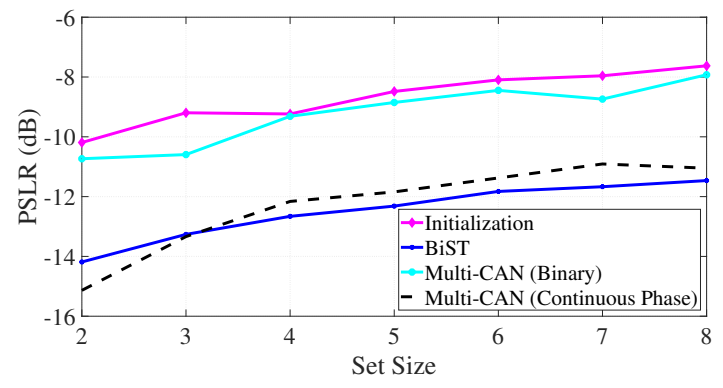

(a) Aperiodic PSL Minimization.

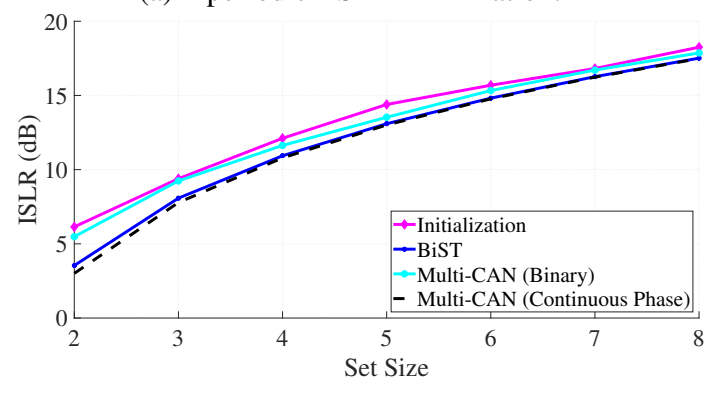

(b) Aperiodic ISL Minimization.

Fig. 6: The effect of the set size $\left(N_{T}\right)$ on the performance of the proposed algorithm: a) PSL minimization. b) ISL minimization.

In Fig. 7, we asses the effect of alphabet size $(L)$ on the performance of the proposed algorithm for the both cases of aperiodic PSL/ISL minimization. Precisely, in the aperiodic case we consider the code length $N=64$, number of transmit antennas $N_{T}=4$, but the alphabet sizes $L=$ $[2,4,8,16,32,64]$. Again, we use 10 independent trials and report the averaged PSLR/ISLR values. The results indicate that in terms of aperiodic PSLR (Fig. 7a), the designed sets of sequences lead to significantly better values while the alphabet size increases. On the other hand, the obtained ISLR values in Fig. $6 \mathrm{~b}$ depicts that the proposed algorithm converges to the lower bound (provided by continuous phased Multi-CAN), when the alphabet size increases. In fact, this figure clearly depicts the superior performance of the proposed algorithm.

\section{E. Comparison with the Other Methods}

In order to compare with the other methods, first notice that all the MM-WeCorr, MM-Corr [7], ISLNew [9], and Direct Iterative Search [10] algorithms, minimize the ISL metric (5), and design sets of sequences with arbitrary phases. These methods, have very close performance to that of the MultiCAN algorithm (meet the lower bound), but with a lower computational complexity which is reported in [7] (except [10] which has a higher computational complexity). However, none of them, meet the lower bound on PSL, specifically when the alphabet size is binary. An example is shown in Fig. 8a, where we have compared the PSLR values of the obtained set of binary sequences with MM-Corr (binary and continous phase) [7] when $N_{T}=3$. A similar comparsion is illustrated in Fig. 8b, but with the ISLNew (binary and continous phase) [9], when $N_{T}=4$. Notice that the binary 


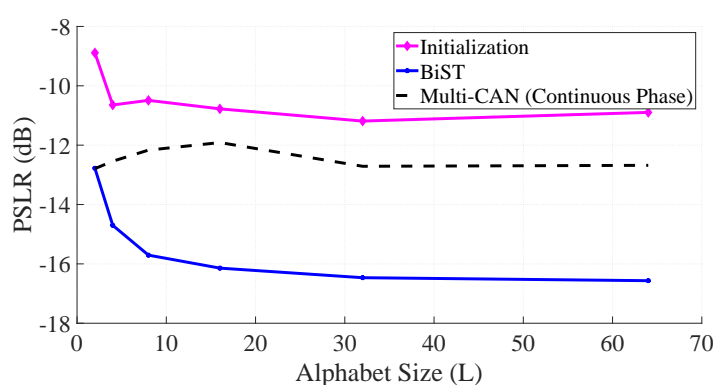

(a) Aperiodic PSL Minimization.

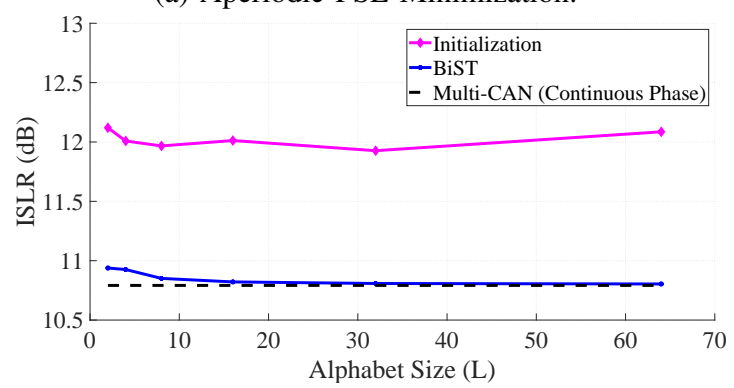

(b) Aperiodic ISL Minimization.

Fig. 7: The effect of the alphabet size $(L)$ on the performance of the proposed algorithm: a) PSL minimization. b) ISL minimization.

set of sequences for the MM-Corr and ISLNew are obtained applying the quantization, as these methods cannot tackle the ISL minimization problem with discrete phase constraint at the design stage. As Fig. 8 illustrates, the set of binary sequences obtained via BiST has better PSLR values in comparison with the set of binary sequences obtained via MM-Corr/ISLNew algorithms.

\section{F. Computational Complexity}

In the sequel, a comparison between the averaged run-time (s) over 10 independent trails for BiST and Multi-CAN algorithms, both designing sets of binary sequences in aperiodic case is reported in TABLE I. The reported values are obtained with a standard PC with Intel (R) Core(TM) i7-600U CPU@ 2.80GHz with installed memory (RAM) $8.00 \mathrm{~GB}$. According to TABLE I, in thesee examples, the computational complexity of the proposed method and Multi-CAN are relatively in a same order. Notice that, even-though MM-Corr, MM-WeCorr and ISLNew have lower computational complexity rather than Multi-CAN (and consequently the proposed BiST algorithm), they don't impose the discrete-phase/binary constraint in the design stage, as opposed to the devised BiST method.

\section{G. The Effect of Designed Sets of Sequences on MIMO Radar System Performance}

Herein, we present performance of the proposed sets of sequences in MIMO radar systems. To this end, we consider a CDM-MIMO radar system with $N_{T}=3$ transmit antennas (Tx), $N_{R}=4$ receive antennas $(\mathrm{Rx})$ in which $M=32$ pulses will send via each transmitter. At receive side, the data cube $L_{c} \times N_{T} \cdot N_{R} \times M$ is available assuming $L_{c}$ range-cells. Let

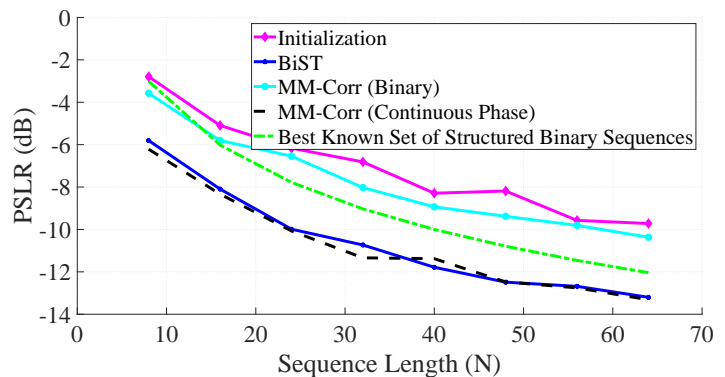

(a) $N_{T}=3$.

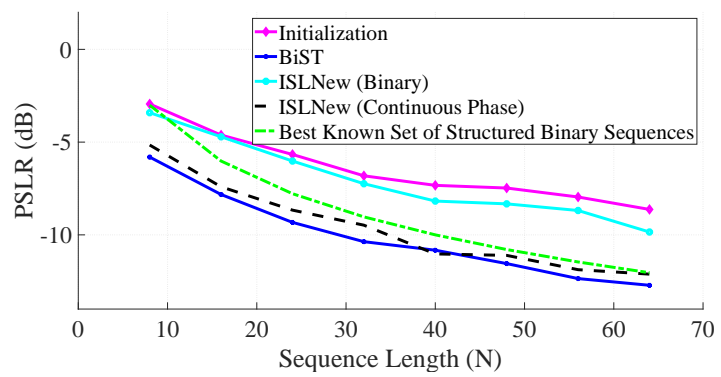

(b) $N_{T}=4$.

Fig. 8: Comparison between the averaged aperiodic PSLR values of the obtained set of sequences via BiST, MM-Corr [7] and ISLNew [9] over 10 independent trails. The number of transmit antennas: a) $N_{T}=3$, b) $N_{T}=4$.

TABLE I: Comparison between the averaged run-time (s) over 10 independent trials for BiST and Multi-CAN, both designing sets of binary sequences.

\begin{tabular}{c|cc|cc|cc}
\hline \hline \multirow{2}{*}{$N$} & \multicolumn{2}{|c}{ Multi-CAN (Binary) } & \multicolumn{2}{c}{ BiST (ISL) } & \multicolumn{2}{c}{ BiST (PSL) } \\
& $N_{T}=3$ & $N_{T}=4$ & $N_{T}=3$ & $N_{T}=4$ & $N_{T}=3$ & $N_{T}=4$ \\
\hline 8 & 0.01 & 0.01 & 0.01 & 0.01 & 0.02 & 0.02 \\
16 & 0.07 & 0.1 & 0.07 & 0.1 & 0.035 & 0.04 \\
24 & 0.2 & 0.25 & 0.2 & 0.3 & 0.2 & 0.25 \\
32 & 0.3 & 0.4 & 0.3 & 0.6 & 0.3 & 0.4 \\
40 & 0.5 & 0.6 & 0.6 & 1.5 & 0.5 & 0.8 \\
48 & 0.8 & 0.9 & 1.2 & 2.6 & 0.9 & 1.1 \\
56 & 1.1 & 1.1 & 2.8 & 4.7 & 2.4 & 3.0 \\
64 & 1.2 & 1.2 & 3.8 & 7.3 & 3.9 & 5.8 \\
\hline \hline
\end{tabular}

us consider a complete receiver processing unit (i. e., matched filter, Doppler and angle processing) for the waveforms emitted by a uniform linear array (ULA)-MIMO radar system with $4 \times 0.5$-wavelength interelement space at $\mathrm{Tx}$ and 0.5 wavelength interelement spacing at $\mathrm{Rx}$ (see [75] for details). In Fig. 9, the range-Doppler plots of the two targets moving toward the radar system is depicted when the code length $N=64$. Fig. 9a shows the case when MIMO radar employs sets of random binary sequences, whereas Fig. 9b illustrates similar situation but emitting sets of binary sequences designed by proposed method (BiST) when minimizing the aperiodic PSL. Two targets assumed to have similar velocities/Doppler frequencies (assumed in Doppler bin 12), but different ranges. The input signal to noise ratio received at the location of the first target is $0 \mathrm{~dB}$ whereas it is $+10 \mathrm{~dB}$ for the second target. The two targets have a separation 10 range-cells, however, as Fig. 9a depicts, just one of the two targets can be correctly detected when the MIMO radar system employs the sets of 


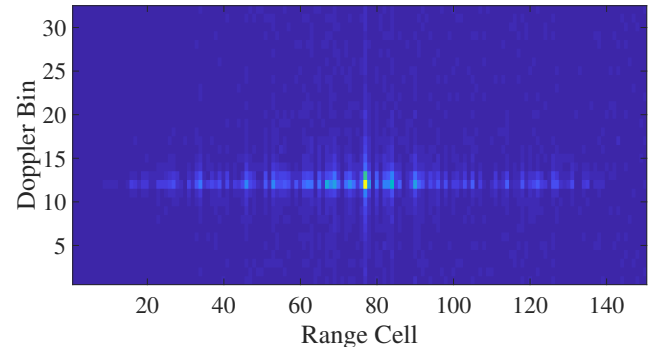

(a) Random.

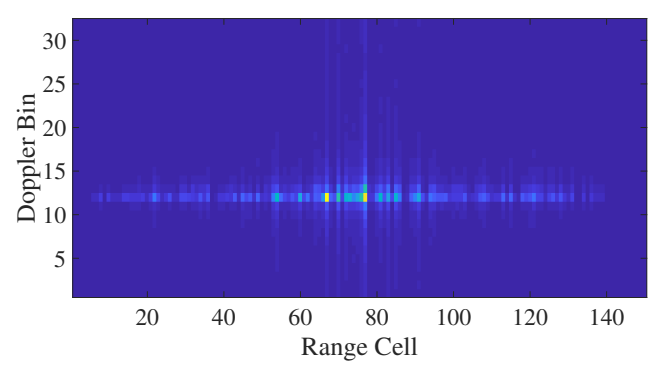

(b) BiST.

Fig. 9: Comparison between range-Doppler separation in a PCM-MIMO radar employing different sets of sequences where a ULA with $N_{T}=3$ and $N_{R}=4$ is used: a) Random set. b) The set obtained by BiST.

random sequences. In fact, the auto- and cross-correlations of the sets of random sequences have led to masking of the weaker target. In contrast, as Fig. 9b illustrates, the two targets can perfectly be discriminated when the proposed sets of sequences is employed. This figure illustrates that the better auto- and cross-correlation properties lead to the better detection.

\section{CONCLUSION}

A computational approach to deal with the problem of sets of sequences design with good aperiodic/periodic auto- and cross-correlation functions for MIMO radar systems has been addressed in this paper. A general framework (called BiST) was devised to tackle the unimodular binary/discrete sets of sequences design problem. The results can be summarized as follows:

- The non-convex and, in general, NP-hard sets of sequences design problem is handled via a novel iterative procedure based on the BCD method.

- Using the DFT technique in designing each code entry of a block in BCD procedure, we minimized a weighted sum of PSL and ISL to design sets of binary/discretephase sequences.

- The provided numerical results confirm that the BiST almost meets the lower bound on ISL while designing binary sets of sequences. Also, the devised algorithm provides sets of binary sequences with better PSL than the structured sets of sequences (i. e., $m$-sequences, Gold, Kasami, etc.).

As future research tracks, it might be interesting to account for the behavior in the Doppler domain of the optimized codes, i.e., tackling the design problem for sets of Doppler-tolerant binary sequences.

\section{APPENDIX A}

RELIANCE OF $P_{d, \boldsymbol{x}_{t}^{(h)}}^{w}$, ON THE OPTIMIZATION VARIABLE

Let $\mathbf{I}_{A}(k)$ and $\mathbf{I}_{B}(k)$ being the indicator functions of sets $A=\{1,2, \ldots, N\}$ and $B=\{-1,-2, \ldots,-N+1\}$ respectively, i.e., $\mathbf{I}_{A}(v)=1$ if $v \in A$, otherwise $\mathbf{I}_{A}(v)=0$.

1) Aperiodic auto- and cross-correlation functions: The cross-correlation function $r_{t l}^{\mathcal{A P}}(k)$ with explicit dependence on $x_{t}(d)$ is,

$$
r_{t l}^{\mathcal{A P}}(k)=a_{d k l}^{\mathcal{A P}} x_{t}(d)+c_{d k l}^{\mathcal{A P}}, k=-N+1, \ldots, N-1 .
$$

with

$$
a_{d k l}^{\mathcal{A P}} \triangleq x_{l}^{*(h)}(d+k) \mathbf{I}_{A}(d+k), k=-N+1, \ldots, N-1 .
$$

and

$$
\begin{aligned}
c_{d k l}^{\mathcal{A P}} \triangleq & \sum_{n=1, n \neq d}^{N-k} x_{t}^{(h)}(n) x_{l}^{*(h)}(n+k) \mathbf{I}_{A}(k+1) \\
& +\sum_{n=-k+1, n \neq d}^{N} x_{t}^{(h)}(n) x_{l}^{*(h)}(n+k) \mathbf{I}_{B}(k) .
\end{aligned}
$$

The aperiodic auto-correlation function $r_{t t}^{\mathcal{A P}}(k)$ is expressed in two different cases:

- The Binary Case $(L=2)$

$$
r_{t t}^{\mathcal{A P}}(k)=a_{d k t}^{\mathcal{A P}} x_{t}(d)+c_{d k t}^{\mathcal{A P}}, k=-N+1, \ldots, N-1 .
$$

where

$$
a_{d k t}^{\mathcal{A P}} \triangleq x_{t}^{(h)}(d+k) \mathbf{I}_{A}(d+k)+x_{t}^{(h)}(d-k) \mathbf{I}_{A}(d-k),
$$

and

$$
\begin{aligned}
c_{d k t}^{\mathcal{A P}} \triangleq & \sum_{n=1, n \neq\{d, d-k\}}^{N-k} x_{t}^{(h)}(n) x_{t}^{(h)}(n+k) \mathbf{I}_{A}(k+1) \\
+ & \sum_{n=-k+1, n \neq\{d, d-k\}}^{N} x_{t}^{(h)}(n) x_{t}^{(h)}(n+k) \mathbf{I}_{B}(k) .
\end{aligned}
$$

- General Discrete Phase $(L \geq 3)$

$$
\begin{aligned}
r_{t t}^{\mathcal{A P}}(k)=a_{d k t}^{\mathcal{A P}} x_{t}(d)+b_{d k t}^{\mathcal{A P}} x_{t}^{*}(d)+c_{d k t}^{\mathcal{A P}}, & \\
& k=-N+1, \ldots, N-1
\end{aligned}
$$

where $^{10}$,

$$
\begin{aligned}
& a_{d k t}^{\mathcal{A P}} \triangleq x_{t}^{*^{(h)}}(d+k) \mathbf{I}_{A}(d+k), \\
& b_{d k t}^{\mathcal{A P}} \triangleq x_{t}^{(h)}(d-k) \mathbf{I}_{A}(d-k),
\end{aligned}
$$

and

$$
\begin{aligned}
c_{d k t}^{\mathcal{A P}} \triangleq & \sum_{n=1, n \neq\{d, d-k\}}^{N-k} x_{t}^{(h)}(n) x_{t}^{*(h)}(n+k) \mathbf{I}_{A}(k+1) \\
& +\sum_{n=-k+1, n \neq\{d, d-k\}}^{N} x_{t}^{(h)}(n) x_{t}^{*(h)}(n+k) \mathbf{I}_{B}(k) .
\end{aligned}
$$

${ }^{10}$ For notational simplicity, the dependency of auto- and cross-correlation to the iteration index $h$ is implicitly assumed and hence omitted. 
2) Periodic auto- and cross-correlation functions: The periodic cross-correlation function with explicit dependence on $x_{t}(d)$ is,

$$
r_{t l}^{\mathcal{P}}(k)=a_{d k l}^{\mathcal{P}} x_{t}(d)+c_{d k l}^{\mathcal{P}}, \quad k=-N+1, \ldots, N-1 .
$$

where

$$
\begin{aligned}
a_{d k l}^{\mathcal{P}} \triangleq x_{l}^{*(h)}(d+k) & \mathbf{I}_{A}(d+k) \\
+x_{l}^{*(h)}(d & +k+N) \mathbf{I}_{A}(d+k+N) \\
& +x_{l}^{*(h)}(d+k-N) \mathbf{I}_{A}(d+k-N),
\end{aligned}
$$

and

$$
\begin{aligned}
c_{d k l}^{\mathcal{P}} \triangleq & \sum_{n=1, n \neq d}^{N}\left[x_{t}^{(h)}(n) x_{l}^{*(h)}(n+k) \mathbf{I}_{A}(n+k)\right. \\
& +x_{t}^{(h)}(n) x_{l}^{*(h)}(n+k+N) \mathbf{I}_{A}(n+k+N) \\
& \left.\quad+x_{t}^{(h)}(n) x_{l}^{*(h)}(n+k-N) \mathbf{I}_{A}(n+k-N)\right] .
\end{aligned}
$$

The periodic auto-correlation function $r_{t t}^{\mathcal{P}}(k)$ is expressed in two different cases:

- The Binary Case $(L=2)$

$$
r_{t t}^{\mathcal{P}}(k)=a_{d k t}^{\mathcal{P}} x_{t}(d)+c_{d k t}^{\mathcal{P}},
$$

where $k=-N+1, \ldots, N-1$,

$$
\begin{aligned}
\quad a_{d k t}^{\mathcal{P}} \triangleq x_{t}^{(h)}(d+k) & \mathbf{I}_{A}(d+k) \\
+x_{t}^{(h)}(d+k-N) & \mathbf{I}_{A}(d+k-N)+x_{t}^{(h)}(d-k) \mathbf{I}_{A}(d-k) \\
& +x_{t}^{(h)}(d-k+N) \mathbf{I}_{A}(d-k+N)
\end{aligned}
$$

with

$$
\begin{aligned}
c_{d k t}^{\mathcal{P}} \triangleq & \sum_{n=1, n \neq\{d, d-k\}}^{N-k} x_{t}^{(h)}(n) x_{t}^{(h)}(n+k) \\
& +\sum_{n=N-k+1, n \neq\{d, d-k+N\}}^{N} x_{t}^{(h)}(n) x_{t}^{(h)}(n+k-N) .
\end{aligned}
$$

- General Discrete Phase $(L \geq 3)$

$$
r_{t t}^{\mathcal{P}}(k)=a_{d k t}^{\mathcal{P}} x_{t}(d)+b_{d k t}^{\mathcal{P}} x_{t}^{*}(d)+c_{d k t}^{\mathcal{P}}
$$

where $k=-N+1, \ldots, N-1$,

$$
\begin{aligned}
a_{d k t}^{\mathcal{P}} \triangleq x_{t}^{*(h)}(d & +k) \mathbf{I}_{A}(d+k) \\
& +x_{t}^{*(h)}(d+k-N) \mathbf{I}_{A}(d+k-N)
\end{aligned}
$$

and

$$
\begin{aligned}
b_{d k t}^{\mathcal{P}} \triangleq x_{t}^{(h)}(d- & k) \mathbf{I}_{A}(d-k) \\
& +x_{t}^{(h)}(d-k+N) \mathbf{I}_{A}(d-k+N)
\end{aligned}
$$

with

$$
\begin{aligned}
c_{d k t}^{\mathcal{P}} \triangleq & \sum_{n=1, n \neq\{d, d-k\}}^{N-k} x_{t}^{(h)}(n) x_{t}^{*(h)}(n+k) \\
& +\sum_{n=N-k+1, n \neq\{d, d-k+N\}}^{N} x_{t}^{(h)}(n) x_{t}^{*(h)}(n+k-N) .
\end{aligned}
$$

\section{REFERENCES}

[1] J. Li and P. Stoica, "MIMO radar with colocated antennas," IEEE Signal Processing Magazine, vol. 24, pp. 106-114, Sept 2007.

[2] B. Friedlander, "Waveform design for MIMO radars," IEEE Transactions on Aerospace and Electronic Systems, vol. 43, pp. 1227-1238, Jul 2007.

[3] C. Y. Chen and P. P. Vaidyanathan, "MIMO radar ambiguity properties and optimization using frequency-hopping waveforms," IEEE Transactions on Signal Processing, vol. 56, pp. 5926-5936, Dec 2008.

[4] H. He, P. Stoica, and J. Li, "Designing unimodular sequence sets with good correlations; including an application to MIMO radar," IEEE Transactions on Signal Processing, vol. 57, pp. 4391-4405, Nov 2009.

[5] H. He, J. Li, and P. Stoica, Waveform design for active sensing systems: a computational approach. Cambridge University Press, 2012.

[6] M. Soltanalian, M. M. Naghsh, and P. Stoica, "A fast algorithm for designing complementary sets of sequences," Signal Processing, vol. 93, pp. 2096 - 2102, Feb 2013.

[7] J. Song, P. Babu, and D. P. Palomar, "Sequence set design with good correlation properties via majorization-minimization," IEEE Transactions on Signal Processing, vol. 64, pp. 2866-2879, June 2016.

[8] Y. Li, S. A. Vorobyov, and Z. He, "Design of multiple unimodular waveforms with low auto- and cross-correlations for radar via majorizationminimization," in 2016 24th European Signal Processing Conference (EUSIPCO), pp. 2235-2239, Aug 2016.

[9] Y. Li and S. A. Vorobyov, "Fast algorithms for designing unimodular waveform(s) with good correlation properties," IEEE Transactions on Signal Processing, vol. 66, pp. 1197-1212, March 2018.

[10] G. Cui, X. Yu, M. Piezzo, and L. Kong, "Constant modulus sequence set design with good correlation properties," Signal Processing, vol. 139, pp. 75-85, 2017.

[11] L. Wu, P. Babu, and D. P. Palomar, "Transmit waveform/receive filter design for MIMO radar with multiple waveform constraints," IEEE Transactions on Signal Processing, vol. 66, pp. 1526-1540, March 2018.

[12] M. Alaee-Kerahroodi, A. Aubry, A. De Maio, M. M. Naghsh, and M. Modarres-Hashemi, "A coordinate-descent framework to design low PSL/ISL sequences," IEEE Transactions on Signal Processing, vol. 65, pp. 5942-5956, Nov 2017.

[13] M. Alaee-Kerahroodi, A. Aubry, A. De Maio, M. M. Naghsh, and M. Modarres-Hashemi, "Design of binary sequences with low PSL/ISL," in 2017 25th European Signal Processing Conference (EUSIPCO), pp. 2211-2215, Aug 2017.

[14] H. Ganapathy, D. A. Pados, and G. N. Karystinos, "New bounds and optimal binary signature sets; part I: Periodic total squared correlation," IEEE Transactions on Communications, vol. 59, pp. 1123-1132, April 2011.

[15] H. Ganapathy, D. A. Pados, and G. N. Karystinos, "New bounds and optimal binary signature sets - part II: Aperiodic total squared correlation," IEEE Transactions on Communications, vol. 59, pp. 14111420, May 2011.

[16] M. A. Abu-Rgheff, Introduction to CDMA wireless communications. Academic Press, 2007.

[17] K. Kettunen, "Code selection for CDMA systems," Department of Information Studies, University of Tampere, Finland, 1997.

[18] Y. Wang and J. P. Coon, "Interference-reducing spreading code design for BS-CDMA with quasi-synchronous reception," in 2010 IEEE 72nd Vehicular Technology Conference - Fall, pp. 1-5, Sept 2010.

[19] S. Y. Sun, H. H. Chen, and W. X. Meng, "A survey on complementarycoded MIMO CDMA wireless communications," IEEE Communications Surveys Tutorials, vol. 17, pp. 52-69, Firstquarter 2015.

[20] S. Y. Sun, H. H. Chen, and W. X. Meng, "A framework to construct three-dimensional complementary codes for multiuser MIMO systems," IEEE Transactions on Vehicular Technology, vol. 64, pp. 2861-2874, July 2015.

[21] J. M. Velazquez-Gutierrez and C. Vargas-Rosales, "Sequence sets in wireless communication systems: A survey," IEEE Communications Surveys Tutorials, vol. 19, pp. 1225-1248, Secondquarter 2017.

[22] "IEEE standard for information technology - telecommunications and information exchange between systems - local and metropolitan networks - specific requirements - part 11: Wireless lan medium access control (MAC) and physical layer (PHY) specifications: Higher speed physical layer (PHY) extension in the $2.4 \mathrm{GHz}$ band," IEEE Std 802.11b-1999, pp. 1-96, Jan 2000.

[23] Z. Liu, Y. L. Guan, and U. Parampalli, "New complete complementary codes for peak-to-mean power control in multi-carrier CDMA," IEEE Transactions on Communications, vol. 62, pp. 1105-1113, March 2014. 
[24] J. Li, P. Stoica, and X. Zheng, "Signal synthesis and receiver design for MIMO radar imaging," IEEE Transactions on Signal Processing, vol. 56, pp. 3959-3968, Aug 2008.

[25] R. Frank, S. Zadoff, and R. Heimiller, "Phase shift pulse codes with good periodic correlation properties," IRE Transactions on Information Theory, vol. 8, pp. 381-382, October 1962.

[26] K. Balachandran, Q. Bi, A. Rudrapatna, J. Seymour, R. Soni, and A. Weber, "Performance assessment of next-generation wireless mobile systems," Bell Labs Technical Journal, vol. 13, pp. 35-58, Winter 2009.

[27] H. He, J. Li, and P. Stoica, Waveform Design for Active Sensing Systems. Cambridge University Press, 2012.

[28] X. Tang and W. H. Mow, "A new systematic construction of zero correlation zone sequences based on interleaved perfect sequences," IEEE Transactions on Information Theory, vol. 54, pp. 5729-5734, Dec 2008.

[29] P. Stoica, H. He, and J. Li, "On designing sequences with impulse-like periodic correlation," IEEE Signal Processing Letters, vol. 16, pp. 703706, Aug 2009.

[30] H. Deng, "Polyphase code design for Orthogonal Netted Radar systems," IEEE Transactions on Signal Processing, vol. 52, pp. 3126-3135, Nov 2004.

[31] L. Zhao, J. Song, P. Babu, and D. P. Palomar, "A unified framework for low autocorrelation sequence design via Majorization-Minimization," IEEE Transactions on Signal Processing, vol. 65, pp. 438-453, Jan 2017.

[32] H. Luke, "Sequences and arrays with perfect periodic correlation," IEEE Transactions on Aerospace and Electronic Systems, vol. 24, pp. 287-294, May 1988

[33] B. Liu, "Orthogonal discrete frequency-coding waveform set design with minimized autocorrelation sidelobes," IEEE Transactions on Aerospace and Electronic Systems, vol. 45, pp. 1650-1657, Oct 2009.

[34] D. J. Rabideau, "MIMO radar waveforms and cancellation ratio," IEEE Transactions on Aerospace and Electronic Systems, vol. 48, pp. 11671178, APRIL 2012

[35] B. Shtarkalev and B. Mulgrew, "Effects of FDMA/TDMA orthogonality on the gaussian pulse train MIMO ambiguity function," IEEE Signal Processing Letters, vol. 22, pp. 153-157, Feb 2015.

[36] K. W. Forsythe and D. W. Bliss, "MIMO radar waveform constraints for GMTI," IEEE Journal of Selected Topics in Signal Processing, vol. 4 pp. 21-32, Feb 2010.

[37] D. J. Rabideau, "Doppler-offset waveforms for MIMO radar," in 2011 IEEE RadarCon (RADAR), pp. 965-970, May 2011.

[38] F. C. Robey, S. Coutts, D. Weikle, J. C. McHarg, and K. Cuomo, "MIMO radar theory and experimental results," in Conference Record of the Thirty-Eighth Asilomar Conference on Signals, Systems and Computers, 2004., vol. 1, pp. 300-304 Vol.1, Nov 2004.

[39] A. Zwanetski and H. Rohling, "Continuous wave MIMO radar based on time division multiplexing," in 2012 13th International Radar Symposium, pp. 119-121, May 2012.

[40] C. Hammes, M. R. B. Shankar, Y. Nijsure, T. Spielmann, and B. Ottersten, "Random phase center motion technique for enhanced angledoppler discrimination using MIMO radars," in 2017 25th European Signal Processing Conference (EUSIPCO), pp. 2221-2225, Aug 2017.

[41] Y. Tang and Y. Lu, "Single transceiver-based time division multiplexing multiple-input-multiple-output digital beamforming radar system: concepts and experiments," IET Radar, Sonar Navigation, vol. 8, pp. 368375, April 2014

[42] H. Sun, F. Brigui, and M. Lesturgie, "Analysis and comparison of MIMO radar waveforms," in 2014 International Radar Conference, pp. 1-6, Oct 2014.

[43] B. M. Popovic, N. Suehiro, and P. Z. Fan, "Orthogonal sets of quadriphase sequences with good correlation properties," IEEE Transactions on Information Theory, vol. 48, pp. 956-959, Apr 2002.

[44] J. Haboba, R. Rovatti, and G. Setti, "Integrated sidelobe level of sets of rotated legendre sequences," in 2011 IEEE International Conference on Acoustics, Speech and Signal Processing (ICASSP), pp. 2632-2635, May 2011.

[45] H. Esmaeili-Najafabadi, M. Ataei, and M. F. Sabahi, "Designing sequence with minimum PSL using chebyshev distance and its application for chaotic MIMO radar waveform design," IEEE Transactions on Signal Processing, vol. 65, pp. 690-704, Feb 2017.

[46] I. A. Arriaga-Trejo, A. G. Orozco-Lugo, and J. Flores-Troncoso, "Design of unimodular sequences with good autocorrelation and good complementary autocorrelation properties," IEEE Signal Processing Letters, vol. 24, pp. 1153-1157, Aug 2017.

[47] S. Imani, M. M. Nayebi, and S. A. Ghorashi, "Colocated MIMO radar SINR maximization under ISL and PSL constraints," IEEE Signal Processing Letters, vol. 25, pp. 422-426, March 2018.
[48] R. Barker, "Group synchronizing of binary digital systems," Communication theory, pp. 273-287, 1953.

[49] C. Nunn and G. Coxson, "Best-known autocorrelation peak sidelobe levels for binary codes of length 71 to 105," IEEE Transactions on Aerospace and Electronic Systems, vol. 44, pp. 392-395, Jan 2008.

[50] T. Cusick and H. Dobbertin, "Some new three-valued cross correlation functions for binary m-sequences," IEEE Transactions on Information Theory, vol. 42, pp. 1238-1240, Jul 1996.

[51] M. Skolnik, Radar Handbook, Third Edition. Electronics electrical engineering, McGraw-Hill Education, 2008.

[52] D. R. Fuhrmann and G. S. Antonio, "Transmit beamforming for MIMO radar systems using signal cross-correlation," IEEE Transactions on Aerospace and Electronic Systems, vol. 44, pp. 171-186, January 2008.

[53] A. Aubry, A. De Maio, and Y. Huang, "PSL-based beampattern design for MIMO radar systems," in 2015 IEEE Radar Conference (RadarCon), pp. 0231-0236, May 2015.

[54] A. Aubry, A. De Maio, and Y. Huang, "MIMO radar beampattern design via PSL/ISL optimization," IEEE Transactions on Signal Processing, vol. 64 , pp. 3955-3967, Aug 2016.

[55] S. Ahmed, J. S. Thompson, Y. R. Petillot, and B. Mulgrew, "Finite alphabet constant-envelope waveform design for MIMO radar," IEEE Transactions on Signal Processing, vol. 59, pp. 5326-5337, Nov 2011.

[56] H. Li and B. Himed, "Transmit subaperturing for MIMO radars with co-located antennas," in 8th European Conference on Synthetic Aperture Radar, pp. 1-4, June 2010.

[57] M. M. Naghsh, M. Modarres-Hashemi, M. Alaee-Kerahroodi, and E. H. M. Alian, "An information theoretic approach to robust constrained code design for MIMO radars," IEEE Transactions on Signal Processing, vol. 65, pp. 3647-3661, July 2017.

[58] T. Naghibi and F. Behnia, "MIMO radar waveform design in the presence of clutter," IEEE Transactions on Aerospace and Electronic Systems, vol. 47, pp. 770-781, Apr 2011.

[59] B. Tang, J. Tang, and Y. Peng, "MIMO radar waveform design in colored noise based on information theory," IEEE Transactions on Signal Processing, vol. 58, pp. 4684-4697, Sept. 2010.

[60] G. Cui, H. Li, and M. Rangaswamy, "MIMO radar waveform design with constant modulus and similarity constraints," IEEE Transactions on Signal Processing, vol. 62, pp. 343-353, Jan 2014.

[61] W. Chen, Z. Cai, R. Chen, and Z. Zhao, "Optimizing polyphase sequences for orthogonal netted radar systems," Journal of Systems Engineering and Electronics, vol. 23, pp. 529-535, Aug 2012.

[62] J. Li, L. Xu, P. Stoica, K. Forsythe, and D. Bliss, "Range compression and waveform optimization for MIMO radar: A cramer rao bound based study," IEEE Transactions on Signal Processing, vol. 56, pp. 218-232, Jan 2008.

[63] K. Forsythe and D. Bliss, "Waveform correlation and optimization issues for MIMO radar," in Conference on Signals, Systems and Computers, pp. 1306-1310, October 2005.

[64] L. Tang, Y. Zhu, and Q. Fu, "Designing waveform sets with good correlation and stopband properties for MIMO radar via the gradientbased method," Sensors, vol. 17, no. 5, p. 999, 2017.

[65] Y. C. Wang, L. Dong, X. Xue, and K. C. Yi, "On the design of constant modulus sequences with low correlation sidelobes levels," IEEE Communications Letters, vol. 16, pp. 462-465, April 2012.

[66] M. Soltanalian and P. Stoica, "Computational design of sequences with good correlation properties," IEEE Transactions on Signal Processing, vol. 60, pp. 2180-2193, May 2012.

[67] J. Bae and N. Goodman, "Widely separated MIMO radar with adaptive waveform for target classification," in 4th IEEE International Workshop on Computational Advances in Multi-Sensor Adaptive Processing (CAMSAP), pp. 21-24, Dec 2011.

[68] Y. Yang and R. Blum, "Minimax robust MIMO radar waveform design," IEEE Journal of Selected Topics in Signal Processing, vol. 1, pp. 147155, June 2007.

[69] Y. Yang and R. Blum, "MIMO radar waveform design based on mutual information and minimum mean-square error estimation," IEEE Transactions on Aerospace and Electronic Systems, vol. 43, pp. 330 343, Jan 2007.

[70] L. Welch, "Lower bounds on the maximum cross correlation of signals (corresp.)," IEEE Transactions on Information Theory, vol. 20, pp. 397 399, May 1974.

[71] M. Soltanalian, M. M. Naghsh, and P. Stoica, "On meeting the peak correlation bounds," IEEE Transactions on Signal Processing, vol. 62, pp. 1210-1220, March 2014.

[72] K. Deb, Multi-objective optimization using evolutionary algorithms, vol. 16. John Wiley \& Sons, 2001. 
[73] S. Boyd and L. Vandenberghe, Convex optimization. Cambridge university press, 2004.

[74] J. Guerci, Cognitive Radar: The Knowledge-Aided Fully Adaptive Approach. Artech House Radar Library, Artech House, 2010.

[75] S. M. Patole, M. Torlak, D. Wang, and M. Ali, "Automotive radars: A review of signal processing techniques," IEEE Signal Processing Magazine, vol. 34, pp. 22-35, March 2017.

[76] S. J. Wright, "Coordinate descent algorithms," Mathematical Programming, vol. 151 , no. 1, pp. 3-34, 2015

[77] A. Aubry, A. De Maio, B. Jiang, and S. Zhang, "Ambiguity function shaping for cognitive radar via complex quartic optimization," IEEE Transactions on Signal Processing, vol. 61, pp. 5603-5619, Nov 2013.

[78] B. Chen, S. He, Z. Li, and S. Zhang, "Maximum block improvement and polynomial optimization," SIAM Journal on Optimization, vol. 22, pp. 87-107, Jan 2012.

[79] Y. K. Han and K. Yang, "New M-ary sequence families with low correlation and large size," IEEE Transactions on Information Theory, vol. 55, pp. 1815-1823, April 2009. 